INPLASY

PROTOCOL

To cite: Zhang et al.

Effectiveness comparisons of various drug interventions the prevention of Etomidate-

Induced myoclonus: a

Bayesian network

meta-analysis. Inplasy protocol

202190087. doi:

10.37766/inplasy2021.9.0087

Received: 24 September 2021

Published: 24 September 2021

Corresponding author:

Kangda Zhang

893892015@qq.com

Author Affiliation:

Huanliang Wang.

Support: National Natural

Science Found.

Review Stage at time of this submission: Piloting of the study selection process.

Conflicts of interest:

None declared.

\section{Effectiveness comparisons of various drug interventions the prevention of Etomidate-Induced myoclonus: a Bayesian network meta-analysis}

Zhang, KD1; Wang, HL2; Wang, LY3; Zhang, DX4; Zhang, ZH5.

Review question / Objective: Multiple therapeutic agents, including opioids, benzodiazepines, ketamine, lidocaine, lowdose etomidate, and dexmedetomidine, are available for the first-line treatment of preventing Etomidate-induced myoclonus. We performed a systematic review and network meta-analysis to compare the efficacy of these treatment strategies.

Information sources: PubMed, Embase, The Cochrane Central Register of Controlled Trials (CENTRAL), and NIH ClinicalTrials.gov will be searched to find relevant articles from January 1977 to August 2021 within the restriction limit of "randomized controlled trial" and "English language". Some English literature from the CNKI database can be supplemented.

INPLASY registration number: This protocol was registered with the International Platform of Registered Systematic Review and Meta-Analysis Protocols (INPLASY) on 24 September 2021 and was last updated on 24 September 2021 (registration number INPLASY202190087).

\section{INTRODUCTION}

Review question / Objective: Multiple therapeutic agents, including opioids, benzodiazepines, ketamine, lidocaine, lowdose etomidate, and dexmedetomidine, are available for the first-line treatment of preventing Etomidate-induced myoclonus. We performed a systematic review and network meta-analysis to compare the efficacy of these treatment strategies.

Condition being studied: Etomidate is a commonly used general anesthetic that provides smooth anesthesia induction conditions with little influence on the respiratory and circulatory systems. However, intravenous bolus administration 
of etomidate is often associated with myoclonus, with a reported incidence of up to $80 \%$ in unpremeditated patients. Myoclonus is a kind of abnormal neuromuscular activity, which can cause damage of muscle fiber, increase the risk of full gastric reflux in patients with aspiration, elevated intraocular pressure resulting in eye vitreous prolapse. Multiple kinds of therapeutic agents are used to prevent Etomidate-induced myoclonus, opioids, ketamine, low-dose etomidate, midazolam, dexmedetomidine, lidocaine were the most studied drugs. However, a comparison of these drugs is unclear on the basis of all the available direct evidence. Some previous meta-analyses have compared the efficacy of only two of the drugs, a comparison of these methods and the optimal option are unclear on the basis of all the available direct evidence. Therefore, it is necessary to carry out an indirect comparison and network metaanalysis to solve clinical problems and guide clinical practice.

\section{METHODS}

Search strategy: Using the combination of MeSH medical subject words and item words, the search terms are combined for literature retrieval through the logical characters "OR" and "AND". Manual retrieval of all detected reviews, metaanalyses, and references included in the research literature.

Participant or population: Inclusion criteria: (1) Adults patients who are purposed to surgical or invasive intervention under etomidate anesthesi. Exclusion criteria:(1) Patients who have the neuropsychological disease, adrenal cortex dysfunction, heart failure, renal, pulmonary, hepatic, or endocrinal diseases, history of allergic reaction to etomidate and other study drugs.(2) Patients who had taken sedative and analgesic drugs on the day of operation. (3)The severity of myoclonus was not graded.

Intervention: Drugs used to prevent Etomidate-induced myoclonus, mainly include opioids, ketamine, low-dose etomidate, midazolam, dexmedetomidine, and lidocaine.

Comparator: A systematic review and network meta-analysis will be performed and the groups will be compared with each other and the best treatment drug will be ranked.

Study designs to be included: Randomized controlled trials (RCTs).

Eligibility criteria: (1) Adults patients who are purposed to surgical or invasive intervention under etomidate anesthesia.(2) Only trials published in English are included.

Information sources: PubMed, Embase, The Cochrane Central Register of Controlled Trials (CENTRAL), and NIH ClinicalTrials.gov will be searched to find relevant articles from January 1977 to August 2021 within the restriction limit of "randomized controlled trial" and "English language". Some English literature from the CNKI database can be supplemented.

Main outcome(s): Incidence of myoclonus after etomidate induction.

Quality assessment / Risk of bias analysis: Two reviewers independently applied the Cochrane Review Manager (Version 5.4 ) to assess the risk of bias of randomized trials, including random sequence generation, allocation concealment, blinding of participants and personnel, blinding of outcome assessment, incomplete outcome data, selective reporting, and other biases. Each trial will be independently by two reviewers and classified to low risk, unclear risk, and high risk.

Strategy of data synthesis: $R$ or STATA software will be used for direct comparisons. Heterogeneity was assessed between studies using the $Q$ test and $I^{2}$ statistic. If there is no significant heterogeneity, a fixed-effect model will be applied to pool data. If significant heterogeneity exists, a random-effects model will be applied. $R$ software will be used to perform the meta-analysis in a 
Bayesian framework. When a closed-loop exists, a node-splitting technique is used to detect consistency.

Subgroup analysis: The intensity of myoclonus was clinically graded as no myoclonus, mild myoclonus, moderate myoclonus, and severe myoclonus. Moderate to severe is of great clinical significance and we consider it as a subgroup. The incidence of moderate and severe myoclonus after etomidate induction will be analyzed.

Sensitivity analysis: Not applicable.

Country(ies) involved: The People's Republic of China.

Keywords: Etomidate - Myoclonus ·

Anesthesia induction - Network metaanalysis.

Contributions of each author:

Author 1 - Huanliang Wang The author will review and revise the manuscript.

Email: timw4788@163.com

Author 2 - Kangda Zhang - The author proposed the research idea, developed a retrieval strategy, and will draft the manuscript.

Email: 893892015@qq.com

Author 3 - Linyu Wang - Author 3 and Author 2 will conduct a literature search, literature selection, and bias risk assessment.

Email: 18846140502@163.com

Author 4 - Danxu Zhang - The author will perform data extraction.

Email: cathy981030@163.com

Author 5 - Zhihua Zhang - The author will perform data extraction.

Email: 654206509@qq.com 